\title{
STUDY AND ANALYSIS OF USER BEHAVIOUR AND USAGE PATTERNS IN A UNIFIED PERSONAL MULTIMEDIA INFORMATION ENVIRIONMENT
}

\author{
Rahul Singh ${ }^{l}$, Juan Camilo Pinzón ${ }^{1}$ \\ Department of Computer Science, San Francisco State University, San Francisco, CA 94132 \\ rsingh@cs.sfsu.edu, juanpin@sfsu.edu
}

\begin{abstract}
The ability to capture information using multiple media simultaneously is becoming common due to the development of powerful and easy to use sensor technologies and consumer products. It has therefore been widely postulated that systems that recognize the semantic correlations between heterogeneous media, model them, and allow interactions across them in unified manners are essential for sustaining effective information assimilation in such true multimedia scenarios. In this context, at the stateof-the-art, a key challenge involves studying and analyzing user behaviors and usage patterns in such information management frameworks. In this paper, we address this issue using an event-based unified multimedia information management system. Our research analyzes, both qualitatively and quantitatively, how users organize, manage, query, explore, and assimilate information in such an environment. We also identify certain key features in the unified model, which are found to be of special value in information access and assimilation. Results from our investigation provide important insights into user behavior in pan-media information organization and interaction frameworks and will be valuable in further development of unified modeling, querying, and interaction techniques.
\end{abstract}

\section{INTRODUCTION}

Till recently, the focus of research in Multimedia was on the development of powerful features to describe media such as images, video, and audio and the use of similarity functions to design user-data interactions (primarily query-retrieval formulations) [10]. This philosophy simplified the multimedia management problem, because a database was assumed to contain only a specific type of media [3]. In a true multimedia setting, such an approach becomes limiting. This is because storing, managing, and querying different types of media separately creates media specific silos and fragments the semantics of the information across them. Thus, recognition and utilization of semantic correlations between heterogeneous media are crucial for developing novel data models and analysis techniques. Such "unified" multimedia approaches can be postulated to provide better (and more natural) user data interaction experiences and improve information assimilation. Given this problem context, three foci for research emerge: (1) Unified multimedia modeling, which deals with the design of generic multimedia data models and data management/processing frameworks that recognize and utilize semantic correlations across heterogeneous media (2) Design of interaction paradigms, which seeks to develop novel display and interaction methodologies, including query-retrieval capabilities, given a unified multimedia framework, and (3) Study and analysis of usage patterns, in unified multimedia environments.

In our recent research $[11,12]$, we proposed a unified multimedia data model based on the notion of an "event", which conceptually corresponds to an observed physical reality parameterized by space and time. An event-based data model is characterized through: (1) Event information, which contains fundamental information at the physical and domain-specific levels related to the event, such as its spatial-temporal attributes, the entities participating in it, and/or domain-specific characteristics. (2) Event relations, which capture connections or associations between events such as aggregation-relationships as well as a variety of spatial, temporal, and spatio-temporal relationships. (3) Media support, which relates the event instance with the heterogeneous media that describe it. Thus, the event-layer serves as a perceptually meaningful bridge across media specific management layers, and allows user-data interactions independent of (but not excluding) specific media type and data sources. Based on the unified eventbased multimedia data model, a personal information management (PIM) system, called eVITAe (acronym for electronic vitae) was proposed by us in [7, 13]. Unlike a multitude of other systems that are directed at this problem (see for instance [1] and references therein), a key feature of eVITAe is its explicit use of an event-based unified approach to data modeling, presentation, interactions, and manipulation. This allows for highly intuitive and experiential interactions [4], not just for high-level operations such as querying and browsing, but also for lowlevel event management operations.

In this paper, using eVITAe, we present a systematic study of user-behavior and usage patterns in an information

\footnotetext{
${ }^{1}$ Equal Contributors
} 
management system that supports unified multimedia modeling, presentation, and interaction. The key contributions of this paper include:

- A systematic study of user behavior, usage patterns, and analysis of information-organization efficacy and user satisfaction in a unified multimedia information management environment. While detailed studies of user behavior in media-rich environments, such as [9] have been conducted earlier, to the best of our knowledge, this is the first attempt to study this issue in the context of unified multimedia modeling.

- Our investigations identify event hierarchies, which describe containment relationships amongst events as a key feature in information search and assimilation. Event hierarchies are found to be nearly as important as time and location, whose importance in information organization is well established [2, 5, 8, 9].

- For purposes of quantification and evaluation, we propose using a combination of metrics that include metrics of access complexity, measurements of user satisfaction, as well as measurement of the cognitive load on users using TLX (NASA Load Index) [15]. This allows for a holistic and multi-faceted evaluation, beyond what is possible using a single metric (such as time spent or number of clicks) or user surveys.

User behavior and patterns observed by us are necessarily influenced by the specific system used. However, many of our experiments were conducted in comparative settings involving other systems that do not employ a unified multimedia model. This allows us to identify the specificities induced by the use of unified multimedia modeling. Furthermore, event-based modeling/processing has increasingly gained popularity, with many approaches, such as $[1,4,5,6,8,9]$ employing this notion explicitly or implicitly. Results from our research can be directly utilized in these contexts.

\section{SYSTEM DESCRIPTION}

The eVITAe event-based information management system consists of three layers that are briefly outlined here. For details we refer the reader to [7, 13]. The Data Layer encompasses the event-based data model and addresses operations pertaining to event and media storage and retrieval. The Core Layer contains the event-level operations and synchronization necessary to support interactions, issue queries and perform automatic operations like spatial-temporal clustering of media to form events. Finally, the Visualization and Interaction Layer supports display and interaction with various semantically relevant event characteristics.

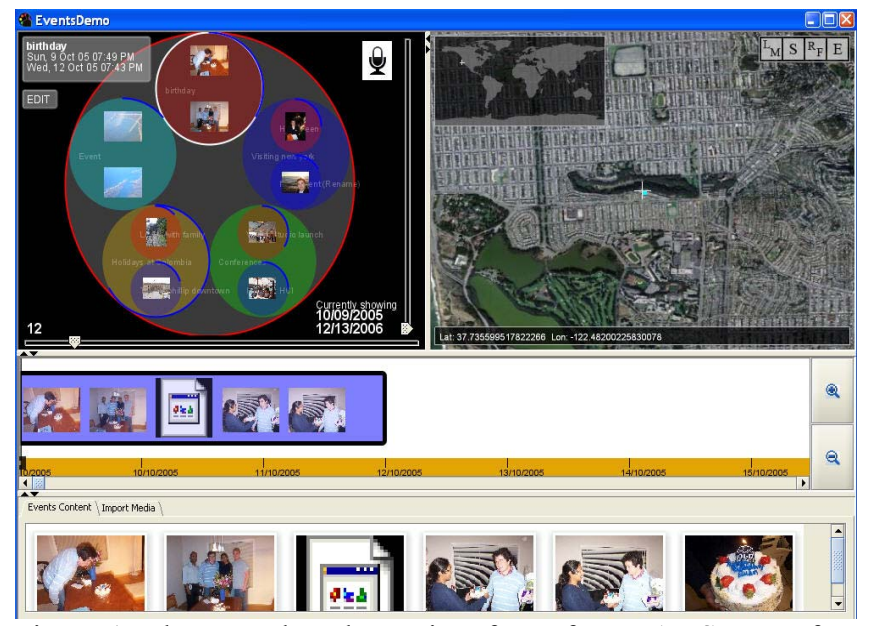

Figure 1: The event-based user interface of eVITAe. Support for information display and interaction is provided through (clockwise from top-left): event-hierarchy panel, spatial information display, temporal information display, media details panel. All operations, including information manipulation, browsing and querying can be supported through intuitive and experiential operations.

Seven modules comprise the Visualization and Interaction layer. These include (see Figure 1): (1) an event hierarchy viewer, (2) an interactive map, (3) an interactive timeline, (4) event detail display, and (5) a media gallery. Additional modules include an audio annotation tool and a universal media player. The system begins by utilizing temporal and spatial information to cluster the media into events. Starting from this point, a user can then operate on the events. This can include, for example, changing/manipulating events by moving specific media between them, creating event hierarchies, and annotating events. Events can be queried by location, time, hierarchical relationships, and metadata. Once an event is selected, the heterogeneous media underlying it is available for perusal, thus allowing unified modeling and interaction.

\section{USER BEHAVIOR AND USAGE PATTERNS}

Several experiments were performed to analyze the usage patterns and to compare this approach with other PIM systems. A combination of three measurement strategies was used as part of these evaluations. These included: (1) Use of questionnaires with a Likert scale to measure subjective attributes (2) Empirical measurements of access complexity through mouse clicks and time, and (3) TLX (NASA Task Load Index), which was used to estimate subjective workload complexity across various environments. TLX is a multi-dimensional rating procedure that provides an overall workload score based on a weighted average of ratings on six subscales: mental demand; physical demand; temporal demand; performance; effort; and frustration. Its definitions and scales are summarized in Table-1. Based on these, the reader may note that lower TLX scores are better. 


\begin{tabular}{|l|l|l|}
\hline Factors & Scale & Descriptions \\
\hline Mental Demand & Low/high & $\begin{array}{l}\text { How much mental and perceptual } \\
\text { activity was required? Was the task } \\
\text { easy or demanding, simple or } \\
\text { complex? }\end{array}$ \\
\hline Physical Demand & Low/high & $\begin{array}{l}\text { How much physical activity was } \\
\text { required? Was the task easy or } \\
\text { demanding, slow or brisk, slack or } \\
\text { strenuous, restful or laborious? }\end{array}$ \\
\hline $\begin{array}{l}\text { Temporal } \\
\text { Demand }\end{array}$ & Low/high & $\begin{array}{l}\text { How much time pressure was felt } \\
\text { due to the rate or pace at which the } \\
\text { tasks or task elements occurred? }\end{array}$ \\
\hline Effort & Low/high & $\begin{array}{l}\text { How hard did you have to work } \\
\text { (mentally and physically) to } \\
\text { accomplish the level of } \\
\text { performance? }\end{array}$ \\
\hline Performance & Good/poor & $\begin{array}{l}\text { How satisfactory was the } \\
\text { experience with the system, in } \\
\text { terms of responsiveness and } \\
\text { stability? }\end{array}$ \\
\hline $\begin{array}{l}\text { Frustration } \\
\text { Level }\end{array}$ & Low/high & $\begin{array}{l}\text { How insecure/discouraged/irritated, } \\
\text { /stressed/annoyed versus secure/, } \\
\text { gratified/content/relaxed/complace } \\
\text { nt did you feel during the task? }\end{array}$ \\
\hline
\end{tabular}

Table 1: TLX definitions and descriptions

The first experiment tracked five users over a period of two months. The amount of data per user and its correlation with the number of events and the complexity of events (as determined by the number of event hierarchies created per user) was measured. The number of media files and the corresponding number of events, per user, is shown in Figure 2 (top row). The complexity of the event-based organization, as defined by the number of event hierarchies is shown in Figure 2 (bottom row). As may be expected, the number of media files directly correlates with the number of events created for each user in four of the five cases, with user-1 being the only exception. Interestingly, most users preferred to create event hierarchies that were reasonably shallow ( 1 or 2 levels deep). For three of the five users, the number of event hierarchies monotonically decreased with depth of the hierarchies. The exceptions were user-3, who had a large number of 2-level event hierarchies and user-1 with a large number of 3-level and 4-level hierarchies. In a survey conducted at the end of the two-month period, all participants listed the hierarchical viewer and timeline as the two most useful information organization tools.

The importance of event hierarchies observed in the first experiment was underlined through another survey of 25 users, who rated on a scale of 1 to 10 , the perceived importance of supporting logical hierarchies in media management. Care was taken to ensure that users did not confuse this notion with that of subdirectory-based file hierarchies. The average score reflecting the importance of supporting logical information hierarchies was 8.7. Based on these results, it can be conjectured that most PIMs, such as photo managers, err by flattening event structures causing the loss of perceptually important information structure.
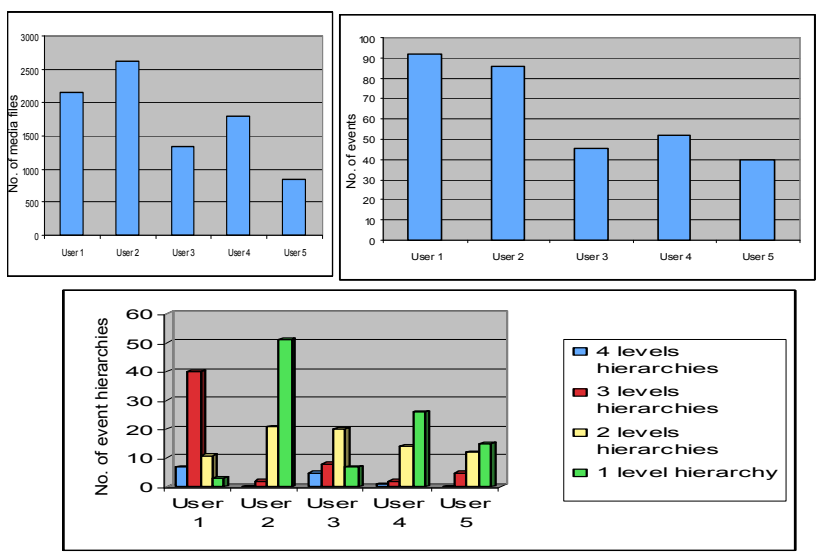

Figure 2: Information organization patterns from Experiment-1 for each user: number of media files (top-left), number of events (topright), distribution of event-hierarchies of varying depths (bottom).

In the second experiment, 10 users (all graduate students) were presented with photographs of their graduation ceremony. In this experiment information access complexity in eVITAe was compared with Memory Miner 1.2 (which primarily supports spatio-temporal interactions) and Windows. The photographs were manually tagged with respective GPS locations so that spatial queries could be performed. Four different queries were then performed by each user. These queries related to locating specific people or events during the ceremony and varied for each user. Users were limited to three minutes for completing each task. If the task was not completed in this timeframe, the corresponding number of clicks and the maximum time of three minutes was recorded for the user-task pair. The order of the queries was alternated using a Latin square to minimize ordering bias. A TLX analysis was performed for each system at the end. The average number of clicks per question, time per-question, and the TLX scores are presented in Figure 3 (the significance based on ANOVA was $\mathrm{F}=4.53$ and $\mathrm{p}=0.05$ ). In terms of information access complexity (clicks and time), eVITAe supporting eventbased access performed favorably. In terms of the TLX score both Memory Miner and Explorer performed poorly when compared with eVITAe. Memory Miner also performed slightly worse than Explorer due primarily to high frustration scores in TLX. Since both eVITAe and Memory Miner support space-time based information access, this experiment highlights the importance of eventbased information organization and its efficacy in information search, beyond using spatial-temporal cues.

In the third experiment, the ability of users to find information satisfying specific information constraints was tested on a dataset that was unfamiliar to each of the participants. The goal here was to study how event-based organization impacted the aforementioned six factors (mental/physical/temporal demand, effort, performance, and frustration) considered in a TLX analysis. 


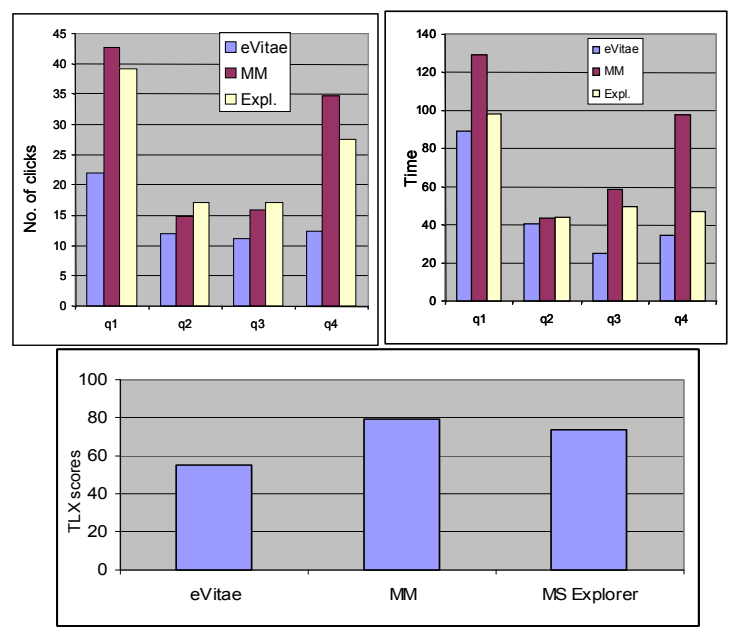

Figure 3: Experimental comparisons with Memory Miner and Windows Explorer in terms of average number of clicks and time per-question (top left and right) and TLX scores (bottom).

As part of this experiment, a comparative study was also performed with Picasa [14] and PhotoMesa. Fourteen queries were given to ten participants. The data set consisted of personal media of one of the authors. The queries were designed to involve temporal, spatial, hierarchical, annotation-based, and perceptual features of the media. Examples of the queries include: "Find any event at the beginning of 2006 that directly caused subsequent events", "Find the photograph of Juan's mother" "What happened immediately after the trip to Mexico?". The biggest contributor to the workload for eVITAe was mental demand. The two largest contributors for Picasa and PhotoMesa were temporal demand and frustration respectively. The TLX values for each factor, the weight given by the users to each factor, and the overall TLX scores, for each of the three applications are presented in Figure 4. The event-based approach in eVITAe, was least demanding, in terms of perceptual load for the user.

\section{CONCLUSIONS}

This paper investigated usage patterns induced by an eventbased unified multimedia data model. We also analyzed specific characteristics of event-based media organization and their impact on efficacy of information access as well as on the cognitive load on users during user-data interactions. These studies identified specific features, such as support for interacting with hierarchical event relationships, to be highly helpful for information assimilation. Insights on how users organize and interact with information in unified multimedia organization models were also obtained. These results are expected to provide valuable insights for researchers engaged in designing models and systems for unified modeling, processing, and analysis of multimedia data as well as those engaged in user-data interaction issues.

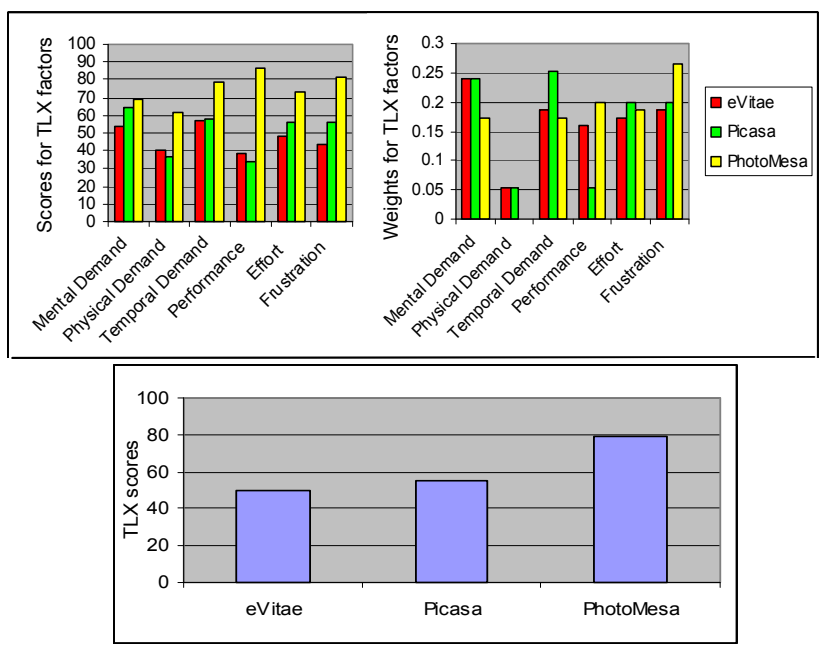

Figure 4: Scores for TLX factors (top left), weights assigned to them (top right), and overall TLX scores (bottom) for information finding/retrieval tasks across eVITAe, Picasa, and PhotoMesa.

\section{REFERENCES}

[1] S. Boll, P. Sandhaus, A. Scherp, and S. Thieme, "MetaXaContext and Content-Driven Metadata Enhancement for Personal Photo Books", ACM MMM, pp. 332- 343, 2007

[2] M. Cooper et al. "Temporal Event Clustering for Digital Photo Collections.”, ACM Trans. on MCCA. pp. 269-288, 2005

[3] J Dionisio, A. Cardenas A., "A Unified Data Model for Representing Multimedia, Timeline, and Simulation Data", IEEE Trans. On KDE, Vol. 10, No. 5, 1998

[4] Jain, R. "Experiential Computing", CACM, 46 (7), 2003

[5] M. Naaman, Y. Song, A Paepcke, H. García-Molina "Automatic Organization for Digital Photographs with Geographic Coordinates”. JDCL 2004.

[6] N. Pattanasri, A. Jatowt, and K. Tanaka, "Enhancing Comprehension of Events in Video through Explanation-onDemand Hypervideo", ACM MMM 2007, pp. 535-544, 2007

[7] J. C. Pinzon, R. Singh, W. Taube, J. Galan, "Designing Interactions In Event-Based Unified Management Of Personal Multimedia Information", ICME, pp. 337 - 340, 2006

[8] Platt J., Czerwinski M., Field B., "PhotoTOC: Automatic Clustering for Browsing Personal Photographs", IEEE Pacific Rim Conference on Multimedia, 2003.

[9] K. Rodden, and K. R. Wood, "How Do People Manage Their Digital Photographs", CHI 2003.

[10] Santini S, Gupta A., "Principles of Schema Design for Multimedia Databases", IEEE Trans. on Multi., 4(2), 2002

[11] R. Singh and R. Jain, "From Information-Centric to Experiential Environments", in Interactive Computation: The New Paradigm, D. Goldin, S. Smolka, and P. Wegner, eds., Springer Verlag, pp. 323 - 351, 2006

[12] R. Singh, Z. Li, P. Kim, D. Pack, R. Jain, "Event-Based Modeling and Processing of Digital Media", CVDB 2004: pp.19-26

[13] R. Singh, R. Knickmeyer, P. Gupta, R. Jain, "Designing experiential environments for management of personal multimedia”. ACM Multimedia, pp. 496-499, 2004.

[14] Picasa: http://www.picasa.com

[15] TLX: http://iac.dtic.mil/hsiac/docs/TLX-UserManual.pdf 